\title{
Effects of Practical Impairments on Cooperative Distributed Antennas Combined with Fractional Frequency Reuse
}

\author{
Jie Zhang ${ }^{\dagger *}$, Rong Zhang*, Xinyi Xu*, Guangjun $\mathrm{Li}^{\dagger}$ and Lajos Hanzo* \\ $\dagger$ School of Comm. and Info. Engineering, UESTC, China. $*$ School of ECS., Univ. of Southampton, UK. \\ Email: 1h@ecs.soton.ac.uk, http://www-mobile.ecs.soton.ac.uk
}

\begin{abstract}
Cooperative Multiple Point (CoMP) transmission aided Distributed Antenna Systems (DAS) are proposed for increasing the received Signal-to-Interference-plus-Noise-Ratio (SINR) in the cell-edge area of a cellular system employing Fractional Frequency Reuse (FFR) in the presence of realistic imperfect Channel State Information (CSI) as well as synchronisation errors between the transmitters and the receivers. Our simulation results demonstrate that the CoMP aided DAS scenario is capable of increasing the attainable SINR by up to $3 \mathrm{~dB}$ in the presence of a wide range of realistic imperfections.
\end{abstract}

\section{INTRODUCTION}

In conventional cellular systems, the classic Unity Frequency Reuse (UFR) pattern may be applied for achieving a high spectral efficiency at the cost of a degraded performance in the cell-edge area due to the severe Co-Channel Interference (CCI) imposed by the neighbouring cells. Hence, the so-called Fractional Frequency Reuse (FFR) pattern has been proposed for improving the received signal strength of cell-edge users at the cost of a reduced spectral efficiency [1]. As a further enhancement, Distributed Antenna Systems (DAS) may be deployed in the cell-edge area of a FFR system in order to further increase the cell-edge Signal-to-Interference-plus-Noise-Ratio (SINR) and providing spatial reuse for compensating for the above-mentioned reduced spectral efficiency.

Naturally, a DAS provides a shorter and hence better link between the Remote Antenna (RA) and the Mobile Stations (MSs) by placing the RA near the cell edge. However, experiments demonstrate that this plain setting of DAS will impose strong CCI emerging from the neighbouring RAs, especially when the MSs are roaming near the angle halfway between the adjacent RAs [2]. A further promising technique of mitigating the RA-induced CCI is constituted by the Multiple-InputMultiple-Output (MIMO) based Cooperative Multiple Point (CoMP) transmissions [3], [4]. Initial studies demonstrate that the cell-edge MSs typically achieve higher SINRs in comparison to both UFR and FFR systems as well as to plain DAS Without the aid of CoMP transmissions.

However, the original CoMP transmission technique requires full Channel State Information (CSI) of all links amongst all BSs and MSs at the transmitter side for approaching the theoretical upper-bound performance. Naturally, the presence of imperfect CSI at the transmitters will erode the efficiency of this CCI mitigation technique. As a result, the cell-edge MSs will benefit from the improved spatial diversity

The financial support of Chinese Scholarship Council, of the RC-UK under the auspices of the India-UK Advanced Technology Centre (IU-ATC), of the EU's Concerto project and that of the EPSRC under the China-UK Science Bridge are gratefully acknowledged.

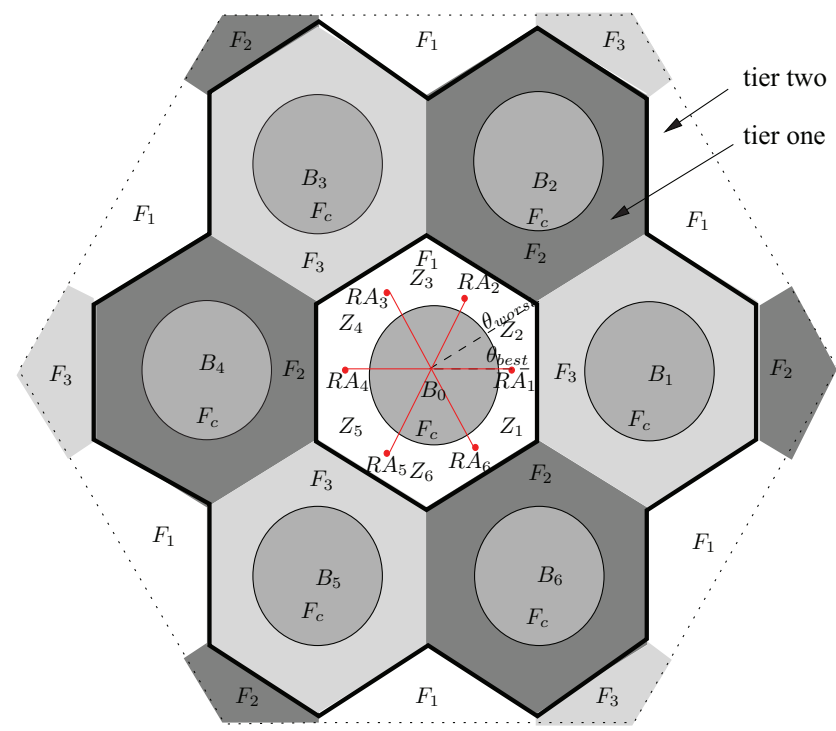

Fig. 1. The cellular topology considered

of all the RAs links, where the specific improvements attained will depend on the accuracy of the CSI available at the transmitter and on the synchronisation errors between the RAs and the MSs.

Hence, our contribution in this paper is to investigate the impact of practical impairments on CoMP aided DAS in the context of a FFR arrangement, including the effects of CSI estimation errors, CSI quantisation errors as well as Orthogonal Frequency Division Multiplexing (OFDM) signal timing and frequency synchronisation errors. To make our investigations as complete as possible, in this paper, we compare the CoMP aided DAS solution to the traditional UFR and FFR transmission regimes as well as to conventional plain DAS dispensing with CoMP.

We organise our paper as follows. In Section II, our system model and assumptions are provided. This is followed by highlighting the benchmarker scenarios of UFR and FFR transmission as well as of conventional DAS dispensing with CoMP. In Section III, we impose a range of practical impairments on CoMP-aided DASs. The system achievable SINR is investigated in Section IV. Finally, we conclude in Section V. 1

\footnotetext{
${ }^{1}$ Notation: Throughout the paper, lower (upper) case boldface letters represent vectors (matrices). The superscripts $(\cdot)^{*},(\cdot)^{T}$ and $(\cdot)^{H}$ denote the conjugate, transpose and conjugate transpose operation, respectively. $\mathbf{E}(\cdot)$ and trace $(\cdot)$ are the expectation and trace operator, respectively. Additionally, $\mathbf{I}_{n}$ represents the $n \times n$ identity matrix.
} 


\section{System DESCRIPTION}

\section{A. Configurations and Assumptions}

Let us first introduce the cellular topology of Fig 1, where 19 hexagonal cellular cells associated with a FFR are employed. As seen in Fig 1 , we let $\mathbb{B}_{O}$ denote the set containing 6 adjacent tier-one cells and 11 tier-two cells, with each having $N_{t}^{B}$ transmit antennas. Within the centred area of a cell shown by the grey circle, MSs are served by the BSs using the frequency set $F_{c}$, while MSs roaming in the cell-edge area are served by RAs using one of the frequencies in the set $\left\{F_{1}, F_{2}, F_{3}\right\}$. Hence, the received SINRs of MSs located near the cell-edge of the conventional cell may be improved as a benefit of their shorter and hence better link between the MSs and the RAs. However, severe CCI still exists when the MSs are located in the vicinity of the direction between two adjacent RAs, which constitutes the worst-case direction.

Against this background, CoMP aided DAS may be employed for mitigating the CCI. Consider a general scenario constituted by $N_{b}$ RAs hosted by the set $\mathbb{B}_{c}$, where each RA is equipped with $N_{t}$ transmit antennas. Let us assume that a total of $N_{u}=N_{b}$ MSs hosted in the set $\mathbb{B}_{u}$ - each equipped with a single receive antenna - are involved in the cooperative transmissions, where each of the $N_{u}$ MSs roams within the coverage area of its anchor RA, as portrayed in Fig. 1. Hence, the scenario may be described by the parameter combination of $\left\{\mathbb{B}_{o}, N_{t}^{B}, N_{b}, N_{t}, N_{u}, N_{r}\right\}$. Furthermore, we let $N_{T}=\left(N_{b} \times N_{t}\right)$ and $N_{R}=\left(N_{u} \times N_{r}\right)$ denote the total number of transmit and receive antennas in the cooperative transmission, respectively. Since we focus on CoMP-aided DAS transmissions, the assumption of a single BS antenna is stipulated for simplicity, while the total power consumption $P_{t}$ is assumed to be the same for the sake of a fair comparison.

Within the arrangement discussed, the assumption of perfect reception of the BSs' data at the RA becomes realistic, when an optical fibre link is used for connecting the RAs and the BS. As a benefit of having a high-bandwidth fibre link between the BS and the RAs, and of the so-called Precise Timing Protocol (PTP) invoked for the synchronisation between the BS and the RAs, the assumption of perfect synchronisation between BS and the RAs becomes reasonable.

\section{B. Benchmarker Scenario}

Before embarking on CoMP-aided and DAS-assisted transmissions, we firstly discuss the classic benchmarker scenarios.

1) Unity Frequency Reuse Scenario: In the UFR scenario, each MS is only served by its own anchor BS, while the remaining active transmissions impose CCI. Hence, the discretetime model of the signal received by MS $j$ may be written as:

$$
y_{j}=\mathbf{h}_{j, j}^{B} \mathbf{t}_{j, j}^{B} s_{j}+\sum_{i \in \mathbb{B}_{o}} \mathbf{h}_{i, j}^{B} \mathbf{t}_{i, i}^{B} s_{i}+n_{j},
$$

where the first two terms represent the desired signal and the CCI imposed by the transmissions of the tier-one and tiertwo cells, which are hosted in the set of $\mathbb{B}_{O}$. The variable $n_{j}$ denotes the circularly symmetric complex Gaussian noise having a covariance of $N_{0}$. Furthermore, $\mathbf{h}_{i, j}^{B} \in \mathbb{C}^{1 \times N_{t}^{B}}$ describes the DownLink (DL) channel between the $i$ th BS and the $j$ th $\mathrm{MS}$, having i.i.d. complex Gaussian entries, while $\mathbf{t}_{i, i}^{B}=\sqrt{P_{t} / N_{t}^{B}}[1,1, \cdots, 1]^{T} \in \mathbb{C}^{N_{t}^{B} \times 1}$ denotes the preprocessing employed at the $i$ th $\mathrm{BS}$ for the sake of transmitting its supported MS's signal. Finally, we let $s_{j}$ denote the transmitted data symbols, which are independent of both the noise and of the channel, obeying $\mathbf{E}\left(s_{j} s_{j}^{*}\right)=1$. Thus, the SINR of the $j$ th MS may be written as:

$$
\gamma_{j}^{u}=\frac{\left|\mathbf{h}_{j, j}^{B} \mathbf{t}_{j, j}^{B} s_{j}\right|^{2}}{\left|n_{j}\right|^{2}+\sum_{i \in \mathbb{B}_{o}}\left|\mathbf{h}_{i, j}^{B} \mathbf{t}_{i, i}^{B} s_{i}\right|^{2}} .
$$

2) Fractional Frequency Reuse Scenario: When FFR is employed in the conventional cellular system, the transmissions destined to the MSs in the centred area are the same as in the UFR scenario. As for the MSs near the cell-edge, the CCI may be substantially decreased because the adjacent cells are potentially assigned different frequency sets for their transmissions, as shown in Fig. 1. As a result, the performance of users roaming near the cell-edge will be improved at the cost of a reduced spectral efficiency. Similarly, the SINR of MS $j$ roaming in the cell-edge area may be written as

$$
\gamma_{j}^{f}=\frac{\left|\mathbf{h}_{j, j}^{B} \mathbf{t}_{j, j}^{B} s_{j}\right|^{2}}{\left|n_{j}\right|^{2}+\sum_{i \in \mathbb{B}_{f}}\left|\mathbf{h}_{i, j}^{B} \mathbf{t}_{i, i}^{B} s_{i}\right|^{2}},
$$

where $\mathbb{B}_{f}$ denotes the subset in $\mathbb{B}_{o}$ using the same frequency set in FFR transmission scenario.

3) DAS-aided FFR scenario: In DAS aided FFR scenario, we assume the total transmit power $P_{t}$ is equally assigned between the RAs for simplicity, and the available power at each RA is denoted as $P_{a}$. In this scenario, $N_{b}$ RAs can use the same channel for supporting more MSs simultaneously, thus the discrete-time model of the signal received by MS $j$ roaming in cell-edge area may be given as

$$
y_{j}=\mathbf{h}_{j, j} \mathbf{t}_{j, j} s_{j}+\sum_{i \in \mathbb{B}_{c,-j}} \mathbf{h}_{i, j} \mathbf{t}_{i, i} s_{i}+\sum_{i \in \mathbb{B}_{f}} \mathbf{h}_{i, j}^{B} \mathbf{t}_{i, i}^{B} s_{i}+n_{j},
$$

where the first term represents the desired signal, while the second and the third term denote the CCI of transmission from other RAs and of transmission within the tier-two cells using the same frequency set, respectively. Moreover, $\mathbf{h}_{i, j} \in \mathbb{C}^{1 \times N_{t}}$ describes the DL channel between the $i$ th RA and the $j$ th MS, obeying i.i.d. complex Gaussian entries, while $\mathbf{t}_{i, i}=$ $\sqrt{P_{a} / N_{t}}[1,1, \cdots, 1]^{T} \in \mathbb{C}^{N_{t} \times 1}$ denotes the preprocessing employed at the $i$ th RA. Similarly, the SINR of MS $j$ roaming in the cell-edge area may be written as

$$
\gamma_{j}^{\text {das }}=\frac{\left|\mathbf{h}_{j, j} \mathbf{t}_{j, j} s_{j}\right|^{2}}{\sum_{i \in \mathbb{B}_{c,-j}}\left|\mathbf{h}_{i, j} \mathbf{t}_{i, i} s_{i}\right|^{2}+\sum_{i \in \mathbb{B}_{f}}\left|\mathbf{h}_{i, j}^{B} \mathbf{t}_{i, i}^{B} s_{i}\right|^{2}+\left|n_{j}\right|^{2}} .
$$

\section{Practical Impairments in COMP-Aided DAS}

\section{A. CoMP-aided DAS with FFR}

In a CoMP scenario, each MS is jointly served by all RAs. Hence, the discrete-time model may be written as:

$$
y_{j}=\mathbf{h}_{j} \mathbf{t}_{j} s_{j}+\sum_{i \in \mathbb{B}_{u,-j}} \mathbf{h}_{j} \mathbf{t}_{i} s_{i}+\sum_{i \in \mathbb{B}_{f}} \mathbf{h}_{i, j}^{B} \mathbf{t}_{i, i}^{B} s_{i}+n_{j},
$$

where the first two terms represent the desired signal and the Multi-User Interference (MUI) imposed by the simultaneous transmissions to other MSs in the cooperative site. The latter contributions are hosted in the set $\mathbb{B}_{u,-j}$. The difference in 
comparison to the system model of the Non-CoMP transmission is that $\mathbf{h}_{j} \in \mathbb{C}^{1 \times N_{T}}$ denotes the joint DL channel between all the $N_{b}$ cooperative RAs and the $j$ th MS, where $\mathbf{h}_{j}=\left[\mathbf{h}_{1, j}, \mathbf{h}_{2, j}, \ldots, \mathbf{h}_{N_{b}, j}\right]$ denotes the joint channel vector. Still referring to Eq. (6), $\mathbf{t}_{j} \in \mathbb{C}^{N_{T} \times 1}$ denotes the joint precoding vector configured for the $j$ th MS of the cooperative site. The third term of Eq. (6) represents the CCI arriving from the tier-two cells, which use the same channel set.

1) Precoding Scheme: In this paper, we employ the linear Joint Signal-to-Leakage-Noise-Ratio (JSLNR) precoding technique [5], which aims for maximising the received signal power at the intended MSs, whilst simultaneously minimising the signal power leaked to other MSs. More specifically, the SLNR $\eta$ at MS $j$ is given by

$$
\eta_{j}=\frac{\operatorname{trace}\left[\mathbf{t}_{j}^{H} \mathbf{h}_{j}^{H} \mathbf{h}_{j} \mathbf{t}_{j}\right]}{\operatorname{trace}\left[\mathbf{t}_{j}^{H}\left(\vartheta / P_{j}+\mathbf{h}_{-j}^{H} \mathbf{h}_{-j}\right) \mathbf{t}_{j}\right]},
$$

with $\vartheta=\left\{N_{r}\left|n_{j}\right|^{2}+\sum_{i \in \mathbb{B}_{f}} \operatorname{trace}\left(\mathbf{t}_{i, i}^{B}{ }^{H} \mathbf{h}_{i, j}^{B}{ }^{H} \mathbf{h}_{i, j}^{B} \mathbf{t}_{i, i}^{B}\right)\right\} \mathbf{I}_{N_{T}}$ and $\mathbf{h}_{-j}=\left[\mathbf{h}_{1}^{T}, \cdots, \mathbf{h}_{j-1}^{T}, \mathbf{h}_{j+1}^{T}, \cdots, \mathbf{h}_{N_{u}}^{T}\right]^{T}$. Hence, the optimisation problem may be stated as

$$
\mathbf{t}_{j}=\arg \max _{\mathbf{t}_{j}} \eta_{j}
$$

It can be seen that the optimisation problem of Eq (8) requires the knowledge of power allocation $P_{j}=$ $\sum_{i=1}^{N_{b}} P_{i, j}$, which has to satisfy the per-RA power constraint $\mathbf{E}\left\{\sum_{j=1}^{N_{u}} \operatorname{trace}\left[\left(\mathbf{t}_{j} x_{j}\right)\left(\mathbf{t}_{j} x_{j}\right)^{H}\right]\right\}=\sum_{j=1}^{N_{u}} \operatorname{trace}\left(\mathbf{t}_{j} \mathbf{t}_{j}^{H}\right) \leq P_{a}$. In this paper, a simple proportional power allocation strategy is advocated:

$$
P_{i, j}=\frac{\operatorname{trace}\left(\mathbf{h}_{i, j} \mathbf{h}_{i, j}^{H}\right)}{\sum_{j=1}^{N_{u}} \operatorname{trace}\left(\mathbf{h}_{i, j} \mathbf{h}_{i, j}^{H}\right)} P_{a} .
$$

Furthermore, the optimisation problem of Eq (8) may be decoupled into the individual optimisation processes by forcing $\mathbf{t}_{j}$ to be an orthonormal matrix as discussed in [5], where we have $\mathbf{t}_{j}=\operatorname{eigv}\left(\mathbf{B}^{-1} A\right)$ representing the eigenvectors corresponding the largest eigenvalue of $\mathbf{B}^{-1} \mathbf{A}$, with $\mathbf{B}=\vartheta / P_{j}+\mathbf{h}_{-j}^{H} \mathbf{h}_{-j}$ and $\mathbf{A}=\mathbf{h}_{j}^{H} \mathbf{h}_{j}$. Finally, the resultant linear precoding matrix $\mathbf{t}_{i, j}$ is multiplied by the allocated power $P_{i, j}$. Hence, the achievable SINR of CoMP-aided DAS transmissions may be expressed as:

$$
\gamma_{j}^{c o m p}=\frac{\left|\mathbf{h}_{j} \mathbf{t}_{j} s_{j}\right|^{2}}{\sum_{i \in \mathbb{B}_{u,-j}}\left|\mathbf{h}_{j} \mathbf{t}_{i} s_{i}\right|^{2}+\sum_{i \in \mathbb{B}_{f}}\left|\mathbf{h}_{i, j}^{B} \mathbf{t}_{i, i}^{B} s_{i}\right|^{2}+\left|n_{j}\right|^{2}}
$$

\section{B. Practical Impairments}

The system performance of CoMP is heavily dependent on the accuracy of the precoding matrix, which is a function of the instantaneous CSI. However, in practice, we are unlikely to have the luxury of perfect CSI both at the receiver and at the transmitter due to the combined effects of CSI estimation errors and the CSI feedback quantisation errors.

1) CSI Estimation Errors: We assume that $\mathrm{MS} j$ is capable of estimating the joint CSI vector $\mathbf{h}_{j}=$ $\left[\mathbf{h}_{1, j}, \mathbf{h}_{2, j}, \cdots, \mathbf{h}_{N_{b}, j}\right] \in \mathbb{C}^{1 \times N_{T}}$, subject to the assumption of a Gaussian CSI estimation error having a variance of $\sigma_{e}^{2}$. Then the channel vector of MS $j$ may be expressed as $\mathbf{h}_{j}=\hat{\mathbf{h}}_{j}+\mathbf{e}_{j}$, where $\mathbf{e}_{j}$ denotes a zero-mean complex Gaussian vector having a variance of $\sigma_{e}^{2}$.
2) CSI Quantisation Errors: After obtaining the estimated CSI, we assume the employment of the Random Vector Quantisation (RVQ) scheme of [6], [7] for quantising the Channel Direction Information (CDI) $\mathbf{h}_{j}=\hat{\mathbf{h}}_{j} /\left\|\hat{\mathbf{h}}_{j}\right\|$, where $\|\cdot\|$ denotes the Euclidean norm and $\left\|\hat{\mathbf{h}}_{j}\right\|$ represents the Channel Quality Information (CQI), which is assumed to be perfectly fed back to the cooperative transmitters. This implies that a CDI quantisation codebook $\mathbf{C}=\left\{\mathbf{c}_{1}, \mathbf{c}_{2}, \ldots, \mathbf{c}_{N_{q}}\right\}$ consisting of $N_{q}=2^{b}$ zero-mean unit-norm complex Gaussian vectors $\mathbf{c}_{i} \in \mathbb{C}^{1 \times N_{T}}$ is constructed and made available to both the MS and to the cooperative transmitter, where $b$ denotes the number of quantisation bits, i.e. codebook index bits. In the quantised feedback regime, the $b$ bits representing the particular codebook index of $\kappa=\max _{i \in\left\{1,2, N_{q}\right\}} \cos \theta, \theta=\angle\left(\hat{\mathbf{h}}_{j}, \mathbf{c}_{i}\right)$ are transmitted, where the codebook may be designed to satisfy diverse design criteria. If we let $\theta$ be the angle between the CDI $\mathbf{h}_{j}$ and the quantisation vector $\mathbf{c}_{\kappa}$, then we have $\mathbf{h}_{j}=\mathbf{c}_{\kappa} \cos \theta+\mathbf{g}_{\kappa} \sin \theta$, where $\mathbf{g}_{\kappa}$ is a unit vector that lies in the null-space of $\mathbf{c}_{\kappa}$. At the transmitter side, these received codebook-index bits are used for regenerating the quantised CSI by combining them with the CQI value.

3) CoMP-aided DAS with imperfect CSI: In the presence of imperfect CSI at the transmitter, the precoding vector $\tilde{\mathbf{t}}_{j}=\arg \max _{\tilde{\mathbf{t}}_{j}} \eta_{j}$ configured for MS $j$ in the CoMP-aided DAS will be designed based on the feedback quantised CSI $\left\{\tilde{\mathbf{h}}_{1}^{T}, \tilde{\mathbf{h}}_{2}^{T}, \cdots, \tilde{\mathbf{h}}_{N_{u}}^{T}\right\}$, where $\tilde{\mathbf{h}}_{j}$ are regenerated by combining the perfectly fedback CQI $\left\|\hat{\mathbf{h}}_{j}\right\|$ and the quantisation index $\kappa$. More specifically, the quantised CSI of MS $j$ is given by $\tilde{\mathbf{h}}_{j}=\left\|\hat{\mathbf{h}}_{j}\right\| \mathbf{c}_{\kappa} \in \mathbb{C}^{1 \times N_{T}}$. Thus, the SINR of MS $j$ associated with imperfect CSI in Eq. (10) has to be modified to

$$
\gamma_{j}^{c o m p, c}=\frac{\left|\mathbf{h}_{j} \tilde{\mathbf{t}}_{j} s_{j}\right|^{2}}{\sum_{i \in \mathbb{B}_{u,-j}}\left|\mathbf{h}_{j} \tilde{\mathbf{t}}_{i} s_{i}\right|^{2}+\sum_{i \in \mathbb{B}_{f}}\left|\mathbf{h}_{i, j}^{B} \mathbf{t}_{i, i}^{B} s_{i}\right|^{2}+\left|n_{j}\right|^{2}} .
$$

\section{Synchronisation Errors}

One of the assumption in above-mentioned transmission scenarios is that the receivers and the transmitters are perfect synchronised, hence the transmitted signal from all transmitters is arrived at the receiver simultaneously. However in reality, when such as an OFDM-based physical layer technique is employed, there may be synchronisation errors due to both the phase-rotation, which is commensurate with the distance travelled between the transmitters and receivers as well as owing to the mismatch of local oscillator frequencies at the transmitter and receiver, which will erode the orthogonality of subcarriers. In this paper, both the time offset and frequency offset are taken into consideration. More explicitly, the demodulated data symbols $y_{j}[l, k]$ at MS $j$ transmitted from RA $j$ of the $l$ th OFDM symbol and subcarrier $k$ under perfect synchronisation can be shown to be

$$
y_{j}[l, k]=\mathbf{h}_{j, j}[l, k] \mathbf{x}_{j, j}[l, k]+n_{j}[l, k],
$$

where $\mathbf{x}_{j, j}[l, k]=\mathbf{t}_{j, j}[l, k] s_{j} \in \mathbb{C}^{N_{t} \times 1}$ denotes the transmitted symbol with $\mathbf{t}_{j, j}[l, k] \in \mathbb{C}^{N_{t} \times 1}$ being the preprocessing at the transmitter, while $\mathbf{h}_{j, j}[l, k] \in \mathbb{C}^{N_{r} \times N_{t}}$ and $n_{j}[l, k]$ are channel transfer function (CTF) of frequency-selective fading channel and the noise at the receiver of the $l$ th symbol and $k$ th subcarrier, respectively. We henceforth omit the subscript $[l, k]$ for simplicity. 
1) Time Offset: When considering the average time offset of $\varepsilon=n_{\varepsilon} T$ between the transmitter and the receiver, where $T$ denotes the sampling duration, Eq. (12) may be expressed as [8]

$$
y_{j}=e^{j 2 \pi(k / N) n_{\varepsilon}} \alpha\left(n_{\varepsilon}\right) \mathbf{h}_{j, j} \mathbf{x}_{j, j}+n_{j}+n_{n_{\varepsilon}},
$$

where $N$ is the Fast Fourier Transform (FFT) size of the OFDM modulation scheme, with the attenuation factor of $\alpha\left(n_{\varepsilon}\right)=\left|\mathbf{h}_{j, j}\right|^{2} \frac{N-n_{\varepsilon}}{N}$ being negligible when the FFT size $N$ is sufficiently high. Moreover, the Inter-SymbolInterference (ISI) imposed by the time offset may be modelled as additional noise $n_{n_{\varepsilon}}$ having a power of

$$
\sigma_{\varepsilon}^{2}=\left|\mathbf{h}_{j, j}\right|^{2}\left[2 \frac{n_{\varepsilon}}{N}-\left(\frac{n_{\varepsilon}}{N}\right)^{2}\right] .
$$

2) Frequency Offset: As far as the frequency offset of $\phi=$ $\frac{\Delta f}{1 / T_{u}}$ is considered with $T_{u}$ representing the data period of one OFDM symbol, the demodulated data symbol in Eq. (12) may be written as [8]

$$
y_{j}=\left\{e^{j \pi \phi} e^{j 2 \pi\left[\left(l N_{s}+N_{g}\right) / N\right] \phi}\right\} \beta(\phi) \mathbf{h}_{j, j} \mathbf{x}_{j, j}+n_{j}+n_{\Omega},
$$

where the attenuation factor of $\beta(\phi)$ may be neglected in the stable state, in which the local offset $\phi$ is usually small. Furthermore, the power of the additional noise $n_{\Omega}$ imposed by the frequency offset may be approximated as

$$
\sigma_{\Omega}^{2} \approx \frac{\pi^{2}}{3} \phi^{2}
$$

When the above-mentioned two types of synchronisation errors coexist, the data symbol may be expressed as $y \approx e^{j 2 \pi(k / N) n_{\varepsilon}} e^{j 2 \pi \phi\left[\left(l T_{u}+T_{g}\right) / T_{u}\right]} \mathbf{h}_{j, j} \mathbf{x}_{j, j}+n_{j}+n_{n_{\varepsilon}}+n_{\Omega}$.

3) CoMP-aided DAS with synchronisation errors: Thus, the SINR of MS $j$, which takes into account both time synchronisation errors and frequency synchronisation errors in our CoMP-based DAS scenario may be expressed as

$$
\gamma_{j}^{c o m p, s} \approx \frac{\left|\mathbf{h}_{j} \mathbf{t}_{j} x_{j}\right|^{2}}{\sum_{i \in \mathbb{B}_{u,-j}}\left|\mathbf{h}_{j} \mathbf{t}_{i} x_{i}\right|^{2}+\sum_{i \in \mathbb{B}_{f}}\left|\mathbf{h}_{i, j}^{B} \mathbf{t}_{i, i}^{B} x_{i}\right|^{2}+\left|n_{t}\right|^{2}},
$$

where $\mathbf{h}_{j}=\left[\mathbf{h}_{1, j}, \mathbf{h}_{2, j}, \cdots, \mathbf{h}_{N_{b}, j}\right]$ denotes the joint channel of MS $j$ while $\left|n_{t}\right|^{2}=\left|n_{j}\right|^{2}+\left|n_{n_{\varepsilon}}\right|^{2}+\left|n_{\Omega}\right|^{2}$ represents the overall noise constituted by the white noise and by the synchronisation errors at receiver.

\section{Performance Evaluation}

In this section, we consider a practical DAS system, consisting of 19 cells, 6 RAs and the correspondent 6 MSs, which may be described by the parameter combination of $\left\{\mathbb{B}_{o}, N_{t}^{B}, N_{b}, N_{t}, N_{u}, N_{r}\right\}=\{19,1,6,1,6,1\}$. The most important simulation parameters are summarised in Table I.

Here, we considered the Urban Micro setup [9], where the inter-cell distance (ISD) and the BS radius were defined as $D=1000 m$ and $R=D / \sqrt{3}$, respectively. The MISO channels of each BS-MS pair are constituted by two components, i.e. by $\mathbf{h}_{i, j}=\left(A_{i, j}^{p l}\right)^{1 / 2} \mathbf{h}_{i, j}^{f}$, where $\mathbf{h}_{i, j}^{f} \in \mathbb{C}^{N_{r} \times N_{t}^{B}}$ represents the fast fading component, which is assumed to be frequencyflat with zero-mean and unity-variance complex Gaussian en-
TABLE I

SIMULATION PARAMETERS CONSIDERED IN THIS PAPER

\begin{tabular}{|l|r|}
\hline Parameters & Value \\
\hline \hline ISD & $1000 \mathrm{~m}$ \\
\hline Carrier Frequency $f_{C}$ & $2.5 \mathrm{GHz}$ \\
\hline System Bandwidth $B=1 / T$ & $15.36 \mathrm{MHz}$ \\
\hline FFT Size N & 1024 \\
\hline CP Length $N_{g}$ & 72 \\
\hline Subcarrier Bandwidth $1 / T_{u}=B / N$ & $15 \mathrm{kHz}$ \\
\hline no. of RA & 6 \\
\hline Est. error $\sigma_{e}$ & 0.1 \\
\hline Pathloss $\left[\alpha^{p l} ; \beta^{p l}\right]$ & {$\left[-3 ; 1.35 \times 10^{7}\right]$} \\
\hline
\end{tabular}

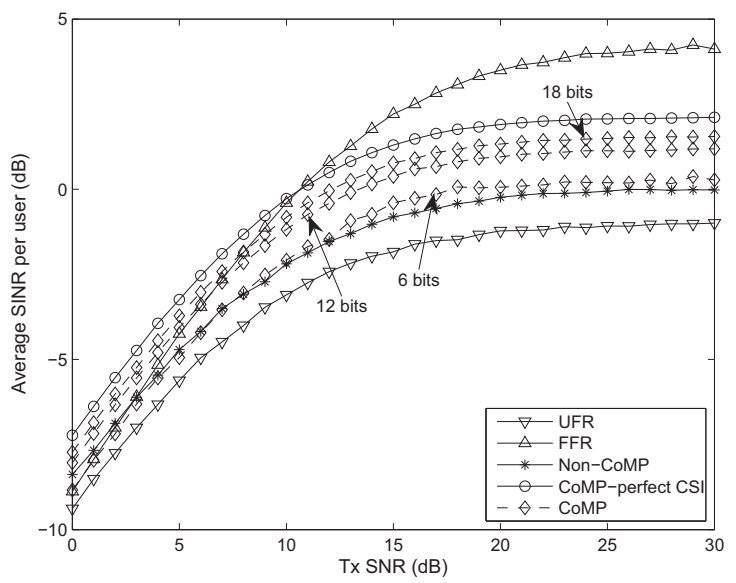

Fig. 2. Achievable SINR per user versus SNR, when the MSs are located in the worst-case direction under both imperfect CSI and synchronisation error

tries, while $A_{i, j}^{p l}=\beta^{p l} d_{i, j}^{\alpha^{p l}}$ describes the pathloss component, where $d_{i, j}$ denotes the distance in meter between the $i$ th BS and $j$ th MS, while we have $\left[\alpha^{p l} ; \beta^{p l}\right]=\left[-3 ; 1.35 \times 10^{7}\right][10]$. The channel between the RA-MS pair is defined similarly. In this paper, we investigate the system performance of MSs which are located in the worst-case direction by 20000 Monte Carlo simulations, when considering the effect of both imperfect CSI and synchronisation errors.

\section{A. Achievable SINR of users in the worst-case direction}

We consider the scenario in which the MSs are located in the angle halfway between the adjacent RAs and experience both imperfect CSI and synchronisation errors. Fig. 2 illustrates the average SINR per user in the worst-case direction of the different transmission arrangements corresponding to three different quantisation bits and time synchronisation offset of $n_{\varepsilon}=0.2 N_{g}$ as well as frequency offset of $\Delta f=0.05$ as a function of $\mathrm{Tx} \mathrm{SNR}^{2}$. It may be observed in Fig. 2 that as expected, the achievable average SINR of all transmission scenarios improves across the entire SNR range spanning from zero to $30 \mathrm{~dB}$. Specifically, the SINR of the FFR scenario is the best at high SNRs as a benefit of having no MUI and a low CCI arriving from tier-two cells. The achievable SINR of CoMP-aided DAS relying on perfect CSI is better than that of the FFR scenario at low SNRs and it also outperforms

${ }^{2}$ SNR here is defined as the transmit power at the transmitter divided by the power of noise at the receiver. 


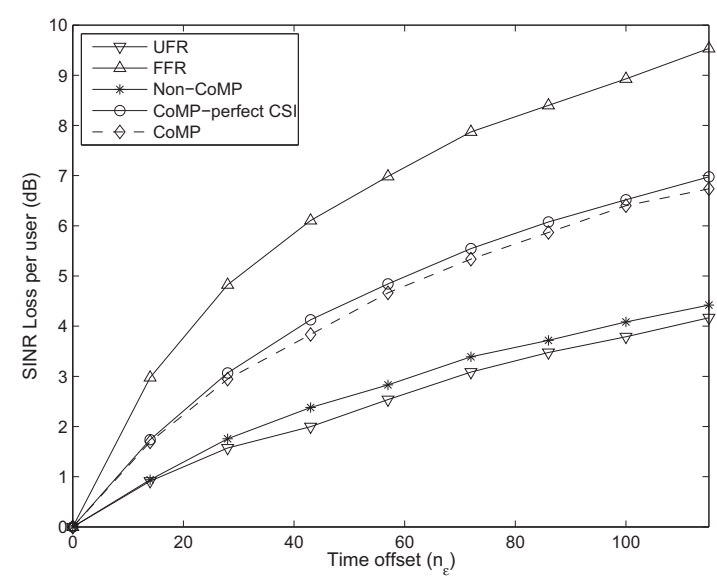

Fig. 3. Average SINR Loss vs. time offset between the transmitter and the receiver without frequency synchronisation errors.

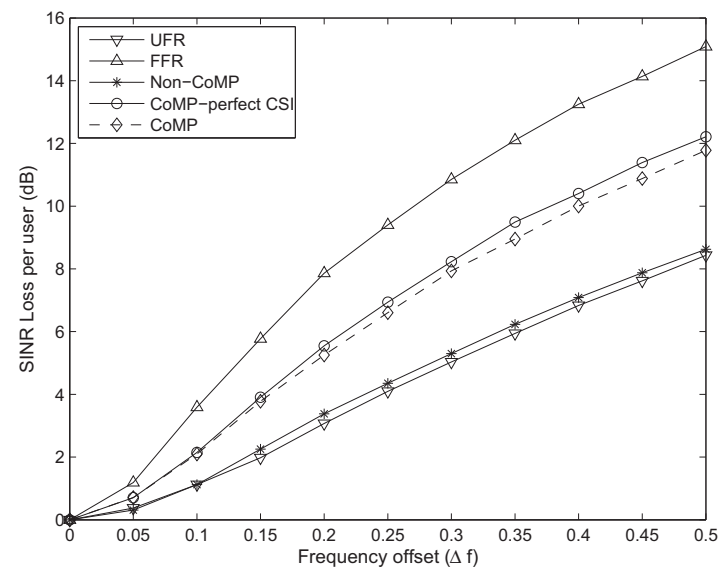

Fig. 4. Average SINR Loss vs. frequency offset between the transmitter and the receiver without time synchronisation errors.

the other two benchmarker scenarios across the entire SNR range. However, in reality we do not have the luxury of perfect CSI either at the receiver or at the transmitter. Hence, the realistically achievable SINRs are degraded, as indicated by the curves marked by the diamond for the imperfect CSI scenario associated with different number of quantisation bits. Naturally, increasing the number of quantisation bits is capable of improving the achievable SINRs. Importantly, even in the presence of imperfect CSI, the achievable SINRs remain superior in comparison to both the plain DAS transmission indicated by the label Non-CoMP and to the UFR transmission. When compared to the FFR scenario, the CoMP-aided DAS provides beneficial SINR improvements at SNRs below 10dB, but performs worse in the high-SNR region, although again, a factor of six spectral efficency improvement was achieved, which is twice as high as that of the UFR scenario.

\section{B. Effects of Time-Offset and Frequency-Offset}

Fig. 3 and Fig. 4 quantify the average SINR loss corresponding to users, which are located in the worst-case direction at an SNR of $20 \mathrm{~dB}$ as a function of both the time-offset and of the frequency-offset, respectively. As for the effects of time synchronisation errors, the SINR loss of all transmission scenarios is increased as the time offset $n_{\varepsilon}$ increased from zero to 115 . Specifically, the SINR loss of the traditional FFR scenario is the highest amongest all other scenarios, since the dominant SINR loss is due to the time-synchronisation errors. The same argument applies to the CoMP-aided DAS, where the SINR loss imposed by time-synchronisation errors is also prominent. On the other hand, since the system performance in both the Non-CoMP aided DAS and in the UFR transmission scenarios is mainly affected by the MUI and CCI, respectively, the SINR loss due to time-synchronisation errors is not obvious. Quantitatively, for both scenarios we have a degradation of no more than $4 \mathrm{~dB}$ owning to time-synchronisation errors. As for the impact of frequency errors, the SINR loss curves exhibit similar trends as in the case of time-synchronisation errors, when the frequency offset increases from zero to $0.5 \Delta f$.

\section{CONCLUSIONS}

We investigated the JSLNR precoding based CoMP-aided DAS in conjunction with FFR under imperfect CSI and synchronisation errors, where a superior received SINR was achieved in comparison to both the plain Non-CoMP DAS transmission, to traditional UFR transmission as well as to FFR transmission at low SNR, when the number of quantisation bits was higher than 12, with the additional benefit of a six-fold improved spectral efficiency in the cell-edge area, when compared to the FFR scenario and a doubled spectral efficiency, when compared to the UFR scenario. Additionally, the sensitivity of the system's performance subjected to synchronisation errors between the transmitter and the receiver was also investigated. Our future work will be related to the optimisation of our proposed CoMP-aided DAS system.

\section{Acknowledgements}

This work is supported by National Natural Science Foundation of China(No. 61006027 and No. 61176025).

\section{REFERENCES}

[1] R. Zhang and L. Hanzo, "Wireless cellular networks," IEEE Vehicular Technology Magazine, vol. 5, no. 4, pp. 31-39, Dec. 2010.

[2] X. Xu, R. Zhang, S. Ghafoor, and L. Hanzo, "Imperfect digitalfiber-optic-link-based cooperative distributed antennas with fractional frequency reuse in multicell multiuser networks," IEEE Trans. Veh. Technol., vol. 60, no. 9, pp. 4439-4449, Nov. 2011.

[3] S. Shamai and B. M. Zaidel, "Enhancing the cellular downlink capacity via co-processing at the transmitting end," in Proc. of 53rd IEEE Vehicular Technology Conference, (VTC' 2001-Spring), Rhodes, Greece, May 2001, pp. 1745-1749.

[4] H. Zhang and H. Dai, "Cochannel interference mitigation and cooperative processing in downlink multicell multiuser MIMO networks," EURASIP Journal on Wireless Communications and Networking, vol. 2004, no. 2, pp. 222-235, Dec. 2004.

[5] M. Sadek, A. Tarighat, and A. H. Sayed, "A leakage-based precoding scheme for downlink multi-user MIMO channels," IEEE Trans. Wireless Commun., vol. 6, no. 5, pp. 1711-1721, May 2007.

[6] W. Santipach and M. L. Honig, "Capacity of a multiple-antenna fading channel with a quantized precoding matrix," IEEE Trans. Inf. Theory, vol. 55, no. 3, pp. 1218-1234, Mar. 2009.

[7] C. K. Au-Yeung and D. J. Love, "On the performance of random vector quantization limited feedback beamforming in a MISO system," IEEE Trans. Wireless Commun., vol. 6, no. 2, pp. 458-462, Feb. 2007.

[8] M. Speth, S. A. Fechtel, G. Fock, and H. Meyr, "Optimum receiver design for wireless broad-band systems using OFDM - Part I," IEEE Trans. Commun., vol. 47, no. 11, pp. 1668-1677, Nov. 1999.

[9] R. Steele and L. Hanzo, Mobile Radio Communications. New York, USA: IEEE Press-John Wiley, 1999.

[10] T. S. Rappaport, Wireless Communications: Principles and Practice. Englewood Cliffs, NJ, USA: Prentice-Hall, 1996. 